\title{
Numerical implementation of the variational formulation for quasi-static brittle fracture
}

\author{
BLAISE BOURDIN ${ }^{\dagger}$ \\ Department of Mathematics, Louisiana State University, \\ Baton Rouge, LA 70803, USA
}

[Received 3 October 2006 and in revised form 6 June 2007]

\begin{abstract}
This paper presents the analysis and implementation of the variational formulation of quasi-static brittle fracture mechanics proposed by G. A. Francfort and J.-J. Marigo in 1998. We briefly present the model itself, and its variational approximation in the sense of $\Gamma$-convergence. We propose a numerical algorithm based on Alternate Minimizations and prove its convergence under restrictive assumptions. We establish a new necessary condition for optimality for the entire time evolution from which we derive the Backtracking algorithm. We give some elements of analysis of the Backtracking algorithm on a simple problem. We present realistic numerical simulations of a traction experiment on a fiber-reinforced matrix, and of the propagation of cracks in a perforated sample under mode-I loading.
\end{abstract}

\section{Introduction}

Fracture mechanics is a very active area of research with vital applications. In recent years, the unexpected collapse of terminal $2 \mathrm{E}$ at Charles de Gaulle airport in France, the disintegration of the Columbia space shuttle upon re-entry, or the crash of American Airlines Flight 582 over Queens, NY were all linked to unexpected fracture. In the area of brittle fracture (which encompass materials as diverse as ceramics, glass, and concrete), many commonly accepted theories, based on Griffith's criterion [Gri21], focus on the propagation of an isolated, pre-existing crack along a given path. In terms of numerical implementation, perhaps the most well-known classes of methods are based on cohesive models and finite elements [XN94, CO96], or on the extended finite element method [MDB99]. The efficiency and versatility of both types of methods have been demonstrated in the literature, although they can also have their weaknesses, including mesh dependency when the crack path is not known beforehand, or difficulty in accounting for initiation and branching.

The work presented here follows an original approach proposed by G. A. Francfort and J.-J. Marigo in [FM98] for quasi-static problems under fixed displacement boundary conditions. Its main virtue is to remain largely compatible with Griffith theory, departing as little as possible to allow crack nucleation, branching, path identification, and interactions between multiple cracks. However, these benefits have a cost in terms of complexity of the numerical implementation. The Francfort-Marigo formulation involves the global minimization of a total energy with respect to any admissible crack set and displacement field, and requires specialized numerical tools which we present in this article. We restrict our numerical experiments to problems simple enough to be rigorously analyzed instead of engaging in very large scale experiments, which is the focus of some pending work.

${ }^{\dagger}$ E-mail: bourdin@math.lsu.edu 
In Section 1, we briefly introduce the Francfort-Marigo model, and detail some of the properties of special interest in view of its numerical implementation. Section 2 is entirely devoted to the description and analysis of the algorithms we introduce: the Alternate Minimizations algorithm is studied in Section 2.3, and the Backtracking algorithm presented in Section 2.4. In Section 3, we conduct a deeper analysis of the Backtracking algorithm, applied to a simple uni-axial traction problem, and present numerical experiments. Lastly, in Section 4, we revisit the fiber pull-out experiment presented in [BFM00, DLM07], and present the numerical simulation of a mode-I experiment on a perforated plate.

\section{Francfort and Marigo's model for quasi-static brittle fracture}

We only briefly recall the essential points of the variational approach to brittle fracture, and refer the reader to the reference above as well as to more recent developments presented in [FL03, DFT04, Cha04, Cha05, BFMar] for a comprehensive presentation and analysis of the model.

In all that follows, we consider an open bounded connected domain $\Omega \subset \mathbb{R}^{N}(N=1,2,3)$ with Lipschitz boundary $\partial \Omega$ representing the crack-free reference configuration of an elastic body. We consider the time interval $[0, T]$ during which we apply a time-dependent displacement boundary condition $g(t ; x)$ on a part $\partial \Omega_{D} \subset \partial \Omega$ with non-null measure, while the remaining part, $\partial \Omega_{N}:=$ $\partial \Omega \backslash \partial \Omega_{D}$, remains traction free. For technical reasons better presented in [BFM00, FL03], we consider an extended domain $\widetilde{\Omega}$ such that $\bar{\Omega} \subset \widetilde{\Omega}$, and an extension (still denoted $g$ ) of the Dirichlet boundary condition to $\widetilde{\Omega}$.

Francfort and Marigo's model relies on two main ingredients: identifying the cracks in a material with the discontinuity set of its displacement field, and-extending Griffith's ideas-deriving the crack evolution from the global minimization of an energy involving competition between bulk and surface terms. The following definition states the form of the kinematically admissible displacement fields, elastic potential, and the total energy for three types of problems.

Definition 1 Let $g$ be such that

$$
g \in L^{\infty}\left([0, T] ; L^{\infty}(\widetilde{\Omega})\right) \cap W^{1,1}\left([0, T] ; H^{1}(\widetilde{\Omega})\right) .
$$

The set $\mathcal{K}_{A}(t)$ of kinematically admissible functions and the elastic potential $W$ are defined as follows:

1. In the elasticity problem, one considers

$$
W(\mathbf{e}(u)):=\frac{1}{2}(\lambda \operatorname{tr} \mathbf{e}(u) \mathbf{I}+2 \mu \mathbf{e}(u)): \mathbf{e}(u),
$$

where $\lambda$ and $\mu$ are the Lamé coefficients of the material considered, $\mathbf{e}(u)$ is the symmetrized gradient of $u$, and ":" represents the dot product of symmetric matrices. The set of kinematically admissible displacements is defined as

$$
\mathcal{K}_{A}(t):=\left\{u \in \operatorname{SBD}(\widetilde{\Omega}): u=g(t) \text { a.e. in } \widetilde{\Omega} \backslash \bar{\Omega} ;\|u\|_{\infty} \leqslant M\right\},
$$

for some given $M$. The role of the constant $M$ is purely technical, and does not affect the practical applications.

2. In the vector-valued $S B V$-problem, $W$ depends on the gradient $\mathbf{D} u$ :

$$
W(\mathbf{D} u):=\mu|\mathbf{D} u|^{2} .
$$


The set of kinematically admissible displacements is defined as

$$
\mathcal{K}_{A}(t):=\{u \in S B V(\widetilde{\Omega}): u=g(t) \text { a.e. in } \widetilde{\Omega} \backslash \bar{\Omega}\} .
$$

3. In the antiplane problem, one considers a cylindrical domain with main axis parallel to the $z$-axis, and deformation along the $z$-axis, independent of the $z$ coordinates. For this class of problems, the displacement field takes the form $u=u_{z} e_{3}$, where $u_{z}$ depends only on $x$ and $y$. The elastic potential becomes

$$
W(\mathbf{e}(u)):=\mu|\mathbf{e}(u)|^{2}=\frac{\mu}{2}\left|\nabla u_{z}\right|^{2},
$$

and the kinematically admissible displacements are given by

$$
\mathcal{K}_{A}(t):=\left\{u_{z} \in S B V(\widetilde{\Omega}): u_{z}=g_{z}(t) \text { a.e. in } \widetilde{\Omega} \backslash \bar{\Omega}\right\} .
$$

Note that depending on the problem, the potential $W$ depends on $\mathbf{e}(u), \mathbf{D} u$ or $\nabla u$. In what follows, we chose to simplify the notations by systematically writing $W(\mathbf{e}(u))$. We trust that this abuse of notation will not cause confusion.

While identifying the crack set and the discontinuity of the displacement field, one has to be careful not to violate an irreversibility principle: displacements can be discontinuous across a given crack, but they do not not have to (consider for example cyclic loads after unloading). However, should a crack exist in the domain at a time $t$, it should also exist at any later time, i.e. cracks are not allowed to heal. The following definition formalizes the relation between both entities:

DEFinition 2 Let $u$ be such that $u(t) \in \mathcal{K}_{A}(t)$ for all $0 \leqslant t \leqslant T$, and $J_{u(t)}$ be the jump set of $u(t)$. The crack set is

$$
\Gamma(u(t)):=\bigcup_{0 \leqslant s \leqslant t} J_{u(s)} .
$$

Notice that $\Gamma(u(t))$ depends on the entire displacement history, i.e. the function $t \mapsto u(t)$, not just the on the displacement at time $t$. Again, we hope that this abuse of notation will not cause confusion.

Definition 3 Let $u$ be such that $u(t) \in \mathcal{K}_{A}(t)$ for all $0 \leqslant t \leqslant T$, and $\Gamma(u(t))$ be as in Definition 2. The total energy associated with $u$ is

$$
E(u(t), \Gamma(u(t)))=\int_{\Omega} W(\mathbf{e}(u(t))) \mathrm{d} x+G_{c} \mathcal{H}^{N-1}\left(\Gamma(u(t)) \backslash \partial \Omega_{N}\right),
$$

where $G_{c}$ denotes the fracture toughness of the material considered. By bulk and surface energies we mean respectively

and

$$
E^{b}(u(t), \Gamma(u(t))):=\int_{\Omega} W(\mathbf{e}(u(t))) \mathrm{d} x
$$

$$
E^{s}(u(t), \Gamma(u(t))):=G_{c} \mathcal{H}^{N-1}\left(\Gamma(u(t)) \backslash \partial \Omega_{N}\right) .
$$

Lastly, and following the viewpoint of [FL03, BFMar], we incorporate the crack growth condition into a global minimization principle for the total energy (1): $u$ is such that for any $0 \leqslant t \leqslant T$, the pair $(u(t), \Gamma(u(t)))$, minimizes

$$
\int_{\Omega} W(\mathbf{e}(v)) \mathrm{d} x+G_{c} \mathcal{H}^{N-1}\left(J_{v} \backslash \partial \Omega_{N}\right)
$$

among all kinematically admissible $v \in \mathcal{K}_{A}(t)$ such that $J_{v} \supset \Gamma(u(t))$. 
As the energy $E$ is non-convex, it can admit local minimizers, the definition of which depends on the topology considered. In the following definition, $A \triangle B$ denotes the symmetric difference of the sets $A$ and $B$.

Definition 4 Let $u$ be such that $u(t) \in \mathcal{K}_{A}(t)$ for all $0 \leqslant t \leqslant T$.

1. $u$ is a local minimizer for $E$ in the sense of the $L^{1}$ norm if and only if there exists $\delta(t)>0$ such that for any $v$ satisfying $v(t) \in \mathcal{K}_{A}(t)$ and $\|u(t)-v(t)\|_{L^{1}(\Omega)} \leqslant \delta(t)$ for $0 \leqslant t \leqslant T$, one has

$$
E(v(t), \Gamma(v(t))) \geqslant E(u(t), \Gamma(u(t))) .
$$

2. $u$ is a local minimizer for $E$ in the sense of the energy norm if and only if there exists $\delta(t)>0$ such that for any $v$ satisfying $v(t) \in \mathcal{K}_{A}(t)$ and $E(u(t)-v(t), \Gamma(u(t)) \triangle \Gamma(v(t))) \leqslant \delta(t)$ for $0 \leqslant t \leqslant T$, one has

$$
E(v(t), \Gamma(v(t))) \geqslant E(u(t), \Gamma(u(t))) .
$$

3. $u$ is a global minimizer for $E$ if and only if for any $v$ such that $v(t) \in \mathcal{K}_{A}(t)$ for $0 \leqslant t \leqslant T$, one has

$$
E(v(t), \Gamma(v(t))) \geqslant E(u(t), \Gamma(u(t))) .
$$

For more details on the analysis of this approach to brittle fracture, we refer the reader to [FL03, DFT04], where existence of global minimizers is proved in the antiplane and vector-valued $S B V$ case. The method used in these proofs involves studying a discrete-in-time version of the problem, and letting the time discretization interval go to zero. In view of the numerical implementation, this implies that we do not have to worry about proving the convergence of the time-discrete model towards the original one.

\subsection{Properties of the Francfort-Marigo energy}

Seeing brittle fracture as the problem of finding global minimizers of (3) remains in large part compatible with Griffith's classical theory of brittle fracture (see [Gri21]). As the crack set at time $t$ is given through a global minimization process among all possible crack states, the FrancfortMarigo model does not require the a priori knowledge of the crack path. It does not require the existence of an initial crack. It does not even assume smooth propagation of cracks (i.e. that the surface energy term is a continuous function of $t$ ). When the surface energy associated to the crack set is not a continuous function of time, we will say that the crack propagates brutally. We recall some properties of $E$ and its minimizers $(u(t), \Gamma(u(t)))$, which are presented in much more detail in [FM98, CGP05]:

1. In the two-dimensional case, if $\mathcal{H}^{N-1}(\Gamma(u(t)))$ is a continuous function of $t$, then $\Gamma(u(t))$ satisfies Griffith's crack propagation criterion.

2. If $g$ is a monotonically increasing load (that is, if $g(t)=t g_{0}$ ), then there exists a critical time $t_{c} \geqslant 0$ such that $\mathcal{H}^{N-1}(\Gamma(u(t)))>0$ for any $t>t_{c}$. In other words, the Francfort-Marigo model will always lead to crack initiation under finite load.

3. Under the same assumptions on $g$ and if the elastic displacement field associated with $g_{0}$ does not have a singularity, then there exists a constant $\delta>0$ such that $\mathcal{H}^{N-1}\left(\Gamma\left(u\left(t_{c}\right)\right)\right)=0$ if $t<t_{c}$ and $\mathcal{H}^{N-1}(\Gamma(u(t)))>\delta$ if $t>t_{c}$. In other words, in the absence of singularity, only brutal crack initiation is possible. 
4. Under the same assumptions on $g$, there exists a total failure load $t_{f}$ such that $E^{b}(u(t), \Gamma(u(t)))=0$ and $E^{s}(u(t), \Gamma(u(t)))=E^{s}\left(u\left(t_{f}\right), \Gamma\left(u\left(t_{f}\right)\right)\right)$ if $t>t_{f}$. For $t>t_{f}$, the minimizers of $E$ correspond to configurations with no elastic energy, and the cracks do not propagate anymore.

Note that points 2 and 3 may seem redundant, but they are not. Point two (from [FM98]) merely states that in the variational model, crack initiation always take place under a finite load (and may be smooth or brutal), whereas point three (adapted from [CGP05]) implies that without singularity, initiation is always brutal.

\section{Numerical implementation}

In order to discretize the Francfort-Marigo functional, one needs to be able to approximate any function in $S B V$ or $S B D$. This is by nature more complicated than building a discrete space allowing jumps across a known curve, and the extended finite element method is not easily applicable. This model also requires the ability to accurately approximate the locations of the cracks, as well as their lengths in two dimensions (and surface in three dimensions), which may not be possible if the cracks are restricted to propagate along edges of faces between elements. Lastly, in light of point 3 above, it is expected that in the absence of singularity in the deformation field, crack initiation will always be brutal. In particular, this means that sensitivities with respect to "small" cracks may never provide a descent direction for the Francfort-Marigo energy, in the case of "brutal" evolution.

\subsection{Approximation by means of elliptic functionals}

Several methods have been proposed, based on discontinuous (see [GP03]) or adaptive (see [BC00, Neg03]) finite elements. The class of methods on which we concentrate here relies on approximating the Francfort-Marigo energy, in the sense of $\Gamma$-convergence, by means of elliptic functionals. It requires introducing a secondary variable $v \in W^{1,2}(\widetilde{\Omega})$, representing the crack in some sense, and extending the brittle fracture energy to

$$
F(u, v):= \begin{cases}E\left(u, J_{u}\right) & \text { if } v \equiv 1, \\ +\infty & \text { otherwise. }\end{cases}
$$

For any $\varepsilon>0$, and $\eta_{\varepsilon} \ll \varepsilon$, define

$$
E_{\varepsilon}(u, v):=\int_{\Omega}\left(v^{2}+\eta_{\varepsilon}\right) W(\mathbf{e}(u)) \mathrm{d} x+G_{c} \int_{\widetilde{\Omega} \backslash \partial \Omega_{N}}\left(\frac{(1-v)^{2}}{4 \varepsilon}+\varepsilon|\nabla v|^{2}\right) \mathrm{d} x,
$$

and

$$
F_{\varepsilon}(u, v):= \begin{cases}E_{\varepsilon}(u, v) & \text { if } u \in W^{1,2}\left(\widetilde{\Omega} \backslash \partial \Omega_{N} ; \mathbb{R}^{q}\right) \text { and } v \in W^{1,2}\left(\widetilde{\Omega} \backslash \partial \Omega_{N}\right), \\ +\infty & \text { otherwise, }\end{cases}
$$

with $q=1$ in the antiplane case, and $q=N$ in the elasticity and vector-valued cases. As for $E$, we will denote by $E_{\varepsilon}^{b}$ and $E_{\varepsilon}^{s}$ the bulk and surface terms in $E_{\varepsilon}$.

This approximation was proposed in [AT90, AT92] for the Mumford-Shah functional, inspired by a now classical example in phase transition [MM77a, MM77b, Alb00] and extended in [Bou98, 
Cha04, Cha05, Gia05]. In the antiplane case, it is known that $F_{\varepsilon} \quad \Gamma$-converges in $L^{2}(\Omega) \times L^{2}(\widetilde{\Omega})$ to $F$ as $\varepsilon \rightarrow 0$, and that the sequence of global minimizers for $F_{\varepsilon}$ is compact. By a classical argument (see for instance [Dal93, Bra02]), this proves that the global minimizers $\left(u^{\varepsilon}, v^{\varepsilon}\right)$ of $F_{\varepsilon}$ converge to that of $F$ (and therefore to that of $E$ ).

\subsection{Time discretization}

A rigorous analysis of the quasi-static evolution problem for the regularized functional has been recently published in [Gia05] in the antiplane case. Again, it is possible to consider a discrete-intime version of the quasi-static evolution where the crack growth hypothesis is enforced at any time step $p$ by minimizing (8) with respect to all admissible $\left(u_{\varepsilon}^{(p)}, v_{\varepsilon}^{(p)}\right)$ such that $v_{\varepsilon}^{(p)} \leqslant v_{\varepsilon}^{(p-1)}$. It has been proved that the time-discrete problem converges to the continuous one when the time discretization goes to 0 , and that the total energy $E_{\varepsilon}\left(u_{\varepsilon}^{(p)}, v_{\varepsilon}^{(p)}\right)$ converges to an absolutely continuous function of $t$.

Finally, the minimization of $F_{\varepsilon}$ at each discrete time step requires a discretization in space. In the antiplane case, one can consider a discretized version $F_{\varepsilon, h}$ of $F_{\varepsilon}$ by means of linear finite elements. Provided that the mesh size $h$ is such that $h \ll \varepsilon$, it is known that $F_{\varepsilon, h} \Gamma$-converges to $F$ (see [BC94, Bou99]). Extending this result to the full elasticity case does not seem to present any difficulty. However, this is outside the scope of this study, and we will take for granted that the restriction $F_{\varepsilon, h}$ of $F_{\varepsilon}$ to discrete functions on a linear finite element space $\Gamma$-converges to $F$ as $\varepsilon \rightarrow 0$ and $h \rightarrow 0$ with $h \ll \varepsilon$.

In order to simplify the implementation of the crack growth condition on a time-discrete scheme, we slightly deviate from the analysis in [Gia05]. Consider a discretization of the time interval [0, T] into $P+1$ time steps $0=t^{(0)}<t^{(1)}<\cdots<t^{(P)}=T$ and $\alpha_{\varepsilon}$ such that $\alpha_{\varepsilon} \rightarrow 0$ as $\varepsilon \rightarrow 0$. At each time step $t^{(p)}, p \geqslant 1$, define

$$
\begin{gathered}
K^{(p-1)}:=\left\{x \in \bar{\Omega}: v^{(p-1)} \leqslant \alpha_{\varepsilon}\right\}, \\
\mathcal{K}_{A}^{(p)}:=\left\{(u, v) \in W^{1,2}\left(\widetilde{\Omega} \backslash \partial \Omega_{N} ; \mathbb{R}^{q}\right) \times W^{1,2}\left(\widetilde{\Omega} \backslash \partial \Omega_{N}\right):\right. \\
\left.u=g\left(t^{(p)}\right) \text { on } \widetilde{\Omega} \backslash \Omega, v=0 \text { on } K^{(p-1)}\right\},
\end{gathered}
$$

and let $\left(u_{\varepsilon}^{(p)}, v_{\varepsilon}^{(p)}\right)$ be a solution of

$$
\min _{(u, v) \in \mathcal{K}_{A}^{(p)}} F_{\varepsilon}(u, v) .
$$

The main difference is that we replace the monotonicity constraint (which in this case would become $v^{(p)} \leqslant v^{(p-1)}$ almost everywhere) by an equality constraint. Doing so allows us to formulate the necessary condition for optimality as equalities instead of inequalities, and to use an unconstrained optimization algorithm. The price we pay for this simplification is that we cannot prove the convergence of the time evolution. Numerical experiments indicate however that our implementation of the irreversibility condition leads to evolutions similar to those obtained while enforcing the monotonicity of $v^{(p)}$ with respect to $p$ (compare for instance the experiment in [BFM00, Sec. 3.2], revisited in Section 4.1, to that presented in [DLM07, Sec. 7.1]).

In the actual numerical implementation, computing global minimizers of $F_{\varepsilon}$ is a major issue, as $F_{\varepsilon}$ is non-convex. Numerical methods in the literature rely on first order necessary conditions 
for optimality. Such methods only ensure convergence to a critical point of $F_{\varepsilon}$, which raises two questions:

1. When working with $F_{\varepsilon}$ for a fixed $\varepsilon>0$, how can we be sure that the critical points we find are local (or global) minima?

2. Can we be sure that these local minima converge as $\varepsilon \rightarrow 0$ to local minima of the $\Gamma$-limit?

The first question is partially answered in Theorem 2 We do not attempt to address the second problem. A similar issue has been settled in [Ton05] for the Modica-Mortola approximation of the perimeter functional.

\subsection{Minimization of $F_{\varepsilon}$ at a given time step}

In the case of the Francfort-Marigo energy, $F_{\varepsilon}$ is quadratic and strictly convex in $u$ and $v$ separately. For a fixed $u$ or $v$, the minimizer of $F_{\varepsilon}(\bullet, v)$ or $F_{\varepsilon}(u, \bullet)$ exists, is unique and can be efficiently computed using a direct method. This property leads naturally to implement the following Alternate Minimizations algorithm, $\delta$ being a small fixed tolerance parameter:

Algorithm 1 (The Alternate Minimizations algorithm)

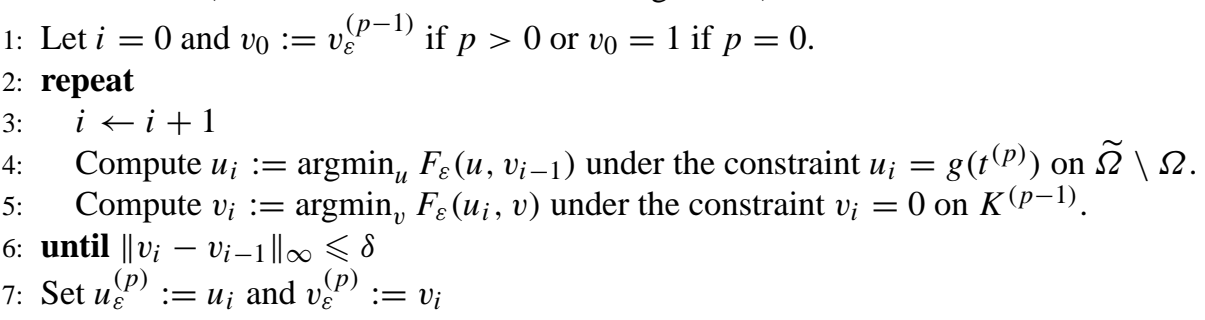

Some elements of convergence of the Alternate Minimizations algorithm follow, under increasingly restrictive hypotheses.

Theorem 1 Let $\left(u_{i}, v_{i}\right)$ be as in Algorithm 1 Then there exists $F_{\varepsilon}^{*} \geqslant 0$ such that

$$
F_{\varepsilon}\left(u_{i}, v_{i}\right) \rightarrow F_{\varepsilon}^{*}
$$

as $i \rightarrow \infty$. Moreover, there exists a critical point $\left(u_{\varepsilon}^{*}, v_{\varepsilon}^{*}\right) \in \mathcal{K}_{A}^{(p)}$ for $F_{\varepsilon}$ such that, up to a subsequence,

$$
\left(u_{i}, v_{i}\right) \stackrel{L^{2}(\Omega) \times L^{2}(\Omega)}{\longrightarrow}\left(u_{\varepsilon}^{*}, v_{\varepsilon}^{*}\right) .
$$

Before proving Theorem 1 we state the following lemma, whose proof is purely algebraic and is not stated here:

LEMma $1 \quad F_{\varepsilon}$ is Gâteaux differentiable at any $(u, v) \in W^{1,2}\left(\widetilde{\Omega} ; \mathbb{R}^{q}\right) \times W^{1,2}(\widetilde{\Omega})$. Considering any $(\tilde{u}, \tilde{v})$ such that $(u+t \tilde{u}, v+t \tilde{v}) \in \mathcal{K}_{A}^{(p)}$ for any small enough $t$, the Gâteaux derivatives of $F_{\varepsilon}$ in the directions $(\tilde{u}, 0)$ and $(0, \tilde{v})$ are given by

$$
\begin{aligned}
\int_{\Omega} \nabla_{u} F_{\varepsilon}(u, v) \tilde{u} \mathrm{~d} x & :=\lim _{t \rightarrow 0} \frac{F_{\varepsilon}(u+t \tilde{u}, v)-F_{\varepsilon}(u, v)}{t} \\
& =\int_{\Omega}\left(v^{2}+\eta_{\varepsilon}\right) \mathbf{D} W(\mathbf{e}(u)): \mathbf{e}(\tilde{u}) \mathrm{d} x,
\end{aligned}
$$

and 


$$
\begin{aligned}
\int_{\widetilde{\Omega}} \nabla_{v} F_{\varepsilon}(u, v) \tilde{v} \mathrm{~d} x & :=\lim _{t \rightarrow 0} \frac{F_{\varepsilon}(u, v+t \tilde{v})-F_{\varepsilon}(u, v)}{t} \\
& =2 \int_{\widetilde{\Omega}}\left\{v W(\mathbf{e}(u))-G_{c}\left(\frac{1-v}{4 \varepsilon}+\varepsilon \Delta v\right)\right\} \tilde{v} \mathrm{~d} x .
\end{aligned}
$$

The proof of this lemma is purely algebraic and does not present any difficulty. For that reason, it is not detailed here.

The expression of the Gâteaux derivatives of $F_{\varepsilon}$ is all we need to prove Theorem 1. Note first that $(12)$ is trivial, on remarking that the sequence $F_{\varepsilon}\left(u_{i}, v_{i}\right)$ is decreasing and bounded from below. The existence of a pair $\left(u_{\varepsilon}^{*}, v_{\varepsilon}^{*}\right)$ such that $\left(u_{i}, v_{i}\right) \stackrel{L^{2}(\Omega) \times L^{2}(\Omega)}{\longrightarrow}\left(u_{\varepsilon}^{*}, v_{\varepsilon}^{*}\right)$ is also easily derived from equi-boundedness in $W^{1,2}$ of the functions $u_{i}$ and $v_{i}$. All that remains to prove is therefore that $\left(u_{\varepsilon}^{*}, v_{\varepsilon}^{*}\right)$ is a critical point of $F_{\varepsilon}$. Since $u_{i} \rightarrow u_{\varepsilon}^{*}$ strongly in $L^{2}$, up to a taking subsequence we have $u_{i} \rightarrow u_{\varepsilon}^{*}$ weakly in $W^{1, p}$. Note also that if $v_{i}$ minimizes $F_{\varepsilon}\left(u_{i}, \bullet\right)$, then using a truncation argument, one finds that $0 \leqslant v_{i} \leqslant 1$ almost everywhere in $\Omega$, from which it follows that

$$
0=\int_{\Omega}\left(v_{i}^{2}+\eta_{\varepsilon}\right) \mathbf{D} W\left(\mathbf{e}\left(u_{i}\right)\right): \mathbf{e}(\tilde{u}) \mathrm{d} x \rightarrow \int_{\Omega}\left(\left(v_{\varepsilon}^{*}\right)^{2}+\eta_{\varepsilon}\right) \mathbf{D} W\left(\mathbf{e}\left(u_{\varepsilon}^{*}\right)\right): \mathbf{e}(\tilde{u}) \mathrm{d} x
$$

as $i \rightarrow \infty$, which is equivalent to

$$
\nabla_{u} F\left(u_{\varepsilon}^{*}, v_{\varepsilon}^{*}\right)=0
$$

Similarly, one obtains

$$
\nabla_{v} F\left(u_{\varepsilon}^{*}, v_{\varepsilon}^{*}\right)=0,
$$

which concludes the proof of Theorem 1 .

Theorem 1 does not rule out cases where several subsequences converge towards different critical points. In the practical implementation of the Alternate Minimizations algorithm, we always observe that the sequence $\left(u_{i}, v_{i}\right)$ converges to a single critical point. We have not been able to prove this, though.

In some cases, we can also prove that the Alternate Minimizations algorithm does indeed converge to the proper critical point. In order to do that, we need to define isolated local minimizers and isolated critical points.

Definition 5 Consider a time step $t^{(p)}$. For any $(u, v) \in \mathcal{K}_{A}^{(p)}$ and any $\alpha>0$, we denote by $B_{\alpha}(u, v)$ the ball of radius $\alpha$ centered at $(u, v)$ :

$$
B_{\alpha}(u, v):=\left\{(\tilde{u}, \tilde{v}) \in \mathcal{K}_{A}^{(p)}:\|(\tilde{u}-u, \tilde{v}-v)\|_{\left(L^{1}\right)^{2}} \leqslant \alpha\right\} .
$$

1. We say that $\left(u_{\varepsilon}, v_{\varepsilon}\right) \in \mathcal{K}_{A}^{(p)}$ is a local minimizer of $F_{\varepsilon}$ if there exists $\delta>0$ such that for any $(u, v) \in B_{\delta}\left(u_{\varepsilon}, v_{\varepsilon}\right)$, one has

$$
F_{\varepsilon}\left(u_{\varepsilon}, v_{\varepsilon}\right) \leqslant F_{\varepsilon}(u, v)
$$

2. We say that $\left(u_{\varepsilon}, v_{\varepsilon}\right) \in \mathcal{K}_{A}^{(p)}$ is an isolated local minimizer of $F_{\varepsilon}$ if there exists $\delta>0$ such that for any $(u, v) \in B_{\delta}\left(u_{\varepsilon}, v_{\varepsilon}\right) \backslash\left\{\left(u_{\varepsilon}, v_{\varepsilon}\right)\right\}$, one has

$$
F_{\varepsilon}\left(u_{\varepsilon}, v_{\varepsilon}\right)<F_{\varepsilon}(u, v) .
$$


3. We say that $\left(u_{\varepsilon}, v_{\varepsilon}\right) \in \mathcal{K}_{A}^{(p)}$ is an isolated critical point of $F_{\varepsilon}$ if there exists $\delta^{\prime}>0$ such that $\left(u_{\varepsilon}, v_{\varepsilon}\right)$ is the only critical point in $B_{\delta^{\prime}}\left(u_{\varepsilon}, v_{\varepsilon}\right)$.

THEOREM 2 Let $\left(u_{i}, v_{i}\right)$ be as in Algorithm 1 , and $\left(u_{\varepsilon}^{*}, v_{\varepsilon}^{*}\right) \in \mathcal{K}_{A}^{(p)}$ be an isolated local minimizer and an isolated critical point for $F_{\varepsilon}$. Then there exists $\eta>0$ such that if $\left\|v_{0}-v_{\varepsilon}^{*}\right\|_{L^{1}} \leqslant \eta$ then

$$
\left(u_{i}, v_{i}\right) \stackrel{L^{2}(\Omega) \times L^{2}(\Omega)}{\longrightarrow}\left(u_{\varepsilon}^{*}, v_{\varepsilon}^{*}\right) .
$$

The proof of this theorem relies on two facts: that $F_{\varepsilon}$ is convex with respect to $u$ and $v$, and that the Alternate Minimizations algorithm yields a sequence with monotonically decreasing energy. Let $\delta$ be admissible for points 2 and 3 in Definition 5 and consider

$$
F_{\delta}:=\inf _{(u, v) \in \partial B_{\delta}\left(u_{\varepsilon}^{*}, v_{\varepsilon}^{*}\right)} F_{\varepsilon}(u, v)
$$

and

$$
K_{\delta}:=\left\{(u, v) \in B_{\delta}\left(u_{\varepsilon}^{*}, v_{\varepsilon}^{*}\right) ; F_{\varepsilon}(u, v) \leqslant \frac{F_{\varepsilon}\left(u_{\varepsilon}^{*}, v_{\varepsilon}^{*}\right)+F_{\delta}}{2}\right\} .
$$

From the isolation hypothesis, $F_{\delta}>F_{\varepsilon}\left(u_{\varepsilon}^{*}, v_{\varepsilon}^{*}\right)$. Observing for example that $t \mapsto F_{\varepsilon}\left((1-t) u_{\varepsilon}^{*}+t u\right.$, $\left.(1-t) v_{\varepsilon}^{*}+t v\right)$ is continuous with respect to $t$ for any $(u, v) \in \mathcal{K}_{A}^{(p)}$, one sees that $K_{\delta}$ is not reduced to $\left(u_{\varepsilon}^{*}, v_{\varepsilon}^{*}\right)$, and that there exists $0<\eta<\delta$ such that $K_{\delta} \supset B_{\eta}\left(u_{\varepsilon}^{*}, v_{\varepsilon}^{*}\right)$. Also, since $F_{\varepsilon}$ is convex with respect to $u$, the function $t \mapsto F_{\varepsilon}\left((1-t) u_{i}+t u_{i+1}, v_{i}\right)$ is decreasing for $0 \leqslant t \leqslant 1$ so that if $\left(u_{i}, v_{i}\right) \in K_{\delta}$, then $\left(u_{i+1}, v_{i}\right) \in K_{\delta}$. Similarly, one finds that $F_{\varepsilon}\left(u_{i+1}, v_{i+1}\right) \in K_{\delta}$, so that the sequence $\left(u_{i}, v_{i}\right)$ converges to some $\left(u^{*}, v^{*}\right) \in K_{\delta}$. Using Theorem 1 we deduce that $\left(u^{*}, v^{*}\right)$ is a critical point, and so $\left(u^{*}, v^{*}\right)=\left(u_{\varepsilon}^{*}, v_{\varepsilon}^{*}\right)$, since $\left(u_{\varepsilon}^{*}, v_{\varepsilon}^{*}\right)$ is the unique critical point of $F_{\varepsilon}$ in $B_{\delta}\left(u_{\varepsilon}^{*}, v_{\varepsilon}^{*}\right)$, which concludes the proof of Theorem 2 .

Theorem 2 requires not only that the local minimizer be isolated, but also that it be an isolated critical point. Indeed, one can imagine a scenario where an isolated local minimizer $\left(u_{\varepsilon}^{*}, v_{\varepsilon}^{*}\right)$ can be approximated by a sequence of critical points $\left(u_{i}, v_{i}\right)$. In this case, for any $\eta>0$, there exists $N>0$ such that $\left(u_{i}, v_{i}\right) \in B_{\eta}\left(u_{\varepsilon}^{*}, v_{\varepsilon}^{*}\right)$ for any $i>N$. Consider now the Alternate Minimizations algorithm initialized with $v_{N}$. Using the convexity of $F_{\varepsilon}$ with respect to $u$, it is easy to see that our algorithm will converge to $\left(u_{N}, v_{N}\right)$ in exactly one iteration...

Fortunately, one can modify the minimization algorithm to avoid such a situation. Upon convergence of the Alternate Minimizations, one can compute the Hessian of $F_{\varepsilon}$ (or its discretization), and check if it admits a negative eigenvalue. If this is the case, one can minimize $F_{\varepsilon}$ along the direction of the associated eigenvector, then resume the Alternate Minimizations algorithm. This modified version of the Alternate Minimizations algorithm still yields a decreasing sequence but can only converge to a local minimizer. For this variant of the Alternate Minimizations algorithm, it is easy to see that Theorem 2 holds even if $\left(u_{\varepsilon}, v_{\varepsilon}\right)$ is not an isolated critical point.

The numerical experiments presented in Sections 3 and 4 rely on the Alternate Minimizations algorithm as described in Algorithm 1 , and do not include this refinement.

\subsection{Necessary optimality condition for the time evolution}

A new necessary condition for optimality of global and local minimizers can be derived from the crack growth condition. This condition can be stated for the regularized or unregularized problems, and the proofs are identical. For brevity, we consider the unregularized problem. 
THEOREM 3 Let $g$ be a monotonically increasing load (i.e. such that $g(t)=\operatorname{tg}(0)$ ). Let $u$ be a global minimizer for $E$. Then for any $t$ and $\tau$ such that $0 \leqslant t \leqslant \tau \leqslant T$, one has

$$
E(u(t), \Gamma(u(t))) \leqslant \frac{t^{2}}{\tau^{2}} E^{b}(u(\tau), \Gamma(u(\tau)))+E^{s}(u(\tau), \Gamma(u(\tau))) .
$$

Proof. Consider any $0 \leqslant \tau \leqslant T$ and suppose that there exists $0 \leqslant s<\tau$ such that

$$
E(u(s), \Gamma(u(s)))>\frac{s^{2}}{\tau^{2}} E^{b}(u(\tau), \Gamma(u(\tau)))+E^{s}(u(\tau), \Gamma(u(\tau))) .
$$

We then construct $v$ given by

$$
v(t):= \begin{cases}u(t) & \text { if } 0 \leqslant t<s, \\ t / \tau u(\tau) & \text { if } s \leqslant t \leqslant \tau .\end{cases}
$$

It is easy to see that $v(t) \in \mathcal{K}_{A}(t)$ for any $0 \leqslant t \leqslant T$, that

$$
\Gamma(v(t))= \begin{cases}\Gamma(u(t)) & \text { if } 0 \leqslant t<s, \\ {\left[\Gamma(u(s)) \backslash J_{u(s)}\right] \cup J_{u(\tau)}} & \text { if } s \leqslant t \leqslant \tau\end{cases}
$$

and that, since $\Gamma(u(s)) \subset \Gamma(u(\tau))$ and $J_{u(\tau)} \subset \Gamma(u(\tau))$,

$$
\begin{aligned}
E(v(s), \Gamma(v(s))) & =\frac{s^{2}}{\tau^{2}} E^{b}(u(\tau), \Gamma(u(\tau)))+G_{c} \mathcal{H}^{N-1}\left(\Gamma(v(s)) \backslash \partial \Omega_{N}\right) \\
& \leqslant \frac{s^{2}}{\tau^{2}} E^{b}(u(\tau), \Gamma(u(\tau)))+G_{c} \mathcal{H}^{N-1}\left(\Gamma(u(\tau)) \backslash \partial \Omega_{N}\right) \\
& <E(u(s), \Gamma(u(s))),
\end{aligned}
$$

which is in contradiction with (6).

REMARK 4 Theorem 3 has interesting consequences in terms of the numerical implementation. Consider a kinematically admissible displacement field $u$, and any $0<\tau<T$. If there exists $0 \leqslant t \leqslant \tau$ such that (18) does not hold, then $u(t)$ is not a global minimizer for $E$ at time $t$, and the function $v$ built in the proof of Theorem 3 has lower total energy.

This remark is the foundation of our Backtracking algorithm as it permits to detect some cases when a displacement field satisfies the optimality conditions with respect to $u$ or $(u, v)$ at each time step, but is not a global minimizer. In the numerical implementation, we use a small parameter $\delta_{\varepsilon}>0$, and at each time step $t^{(p)}$ we check if (18) is verified within a tolerance $\delta_{\varepsilon}$ for all $t^{(r)}$ with $0<r<p$. If it is not, we return to step $r$, initializing the Alternate Minimizations algorithm with $v^{(p)}$.

ALGORITHM 2 (The Backtracking algorithm)

1: $v_{0} \leftarrow 1$

2: repeat

Compute $\left(u^{(p)}, v^{(p)}\right)$ using the Alternate Minimizations algorithm initialized with $v_{0}$.

4: $\quad$ Compute the bulk energy $E_{\varepsilon}^{b}\left(u^{(p)}, v^{(p)}\right)$ and the surface energy $E_{\varepsilon}^{s}\left(u^{(p)}, v^{(p)}\right)$. 


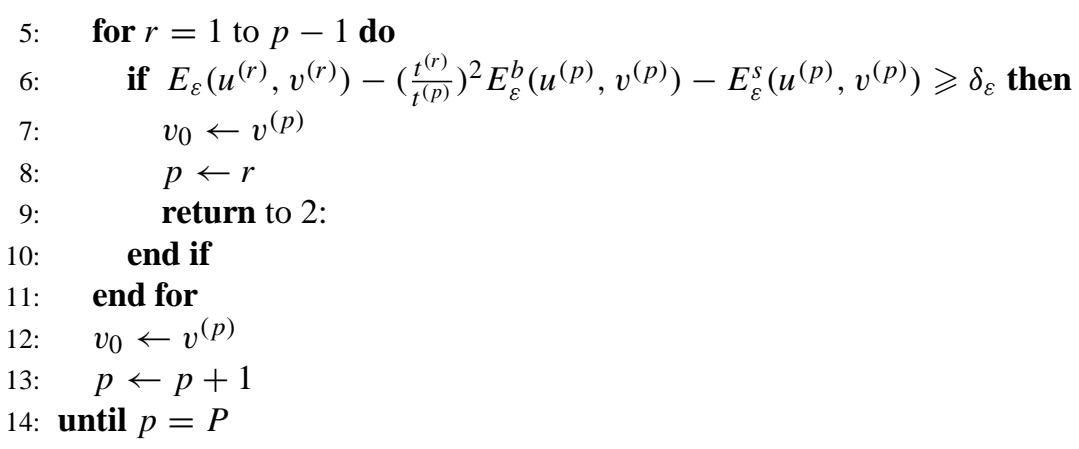

Algorithm 2 raises two questions: does the Backtracking activate, and if so, does it terminate, i.e. what prevents it from entering an infinite loop? These questions require a better understanding of the stability of the critical points of $F_{\varepsilon}$ and we can only provide partial answers. What is clear is that if Algorithm 2 terminates, then it generates a family $\left(u^{(p)}, v^{(p)}\right)$ such that $\left(u^{(p)}, v^{(p)}\right)$ is a critical point of $F_{\varepsilon}$ for any $0 \leqslant p \leqslant P$, which also satisfies the necessary optimality condition with respect to time given in Theorem 3

Of course, the Backtracking algorithm cannot activate unless the minimization algorithm detects new critical points. Numerical experiments suggest that as the load increases, some local minimizers evolve into saddle points, a factor leading to a bifurcation of the Alternate Minimizations algorithm and the discovery of new critical points. A rigorous analysis of this phenomenon can be conducted in a simple unidimensional case.

\section{Some elements of analysis of the Backtracking algorithm}

Consider a two- or three-dimensional beam occupying the domain $\Omega=(-l, l) \times \omega$, where $|\omega|=$ $1 \ll l$, and with elastic coefficients $E=1$ and $v=0$. At any time $t>0$, both ends of the beam are subject to a uniform displacement $u=-t$ on $\{-l\} \times \omega$ and $u=t$ on $\{l\} \times \omega$. Assuming that the deformation and crack fields depend only on the first variable, problem (1) reduces to finding, for any $t>0, u(t) \in S B V(-l, l)$ such that $u(t)=-t$ at $x=-l, u(t)=t$ at $x=l, J_{u(t)} \supset J_{u(s)}$ for any $s<t$, minimizing

$$
\frac{1}{2} \int_{-l}^{l}\left(u(t)^{\prime}\right)^{2} \mathrm{~d} x+G_{c} \#\left(J_{u(t)}\right)
$$

where \# represents the counting measure.

For this problem, it is known (see [FM98]) that there exists a critical load $t=t_{c}=\sqrt{G_{c} l}$ such that if $t<t_{c}$, the global minimizer of (19) corresponds to the uncracked state with $u^{e}(x)=t x / l$. For loads $t>t_{c}$ the global minimum is reached by any function $u^{f}(x)=-t$ for $x<x_{c}$ and $u^{f}(x)=t$ for $x>x_{c}, x_{c}$ being any point in $(-l, l)$.

Under the assumptions above, the regularized energy $E_{\varepsilon}$ can be written as

$$
E_{\varepsilon}(u, v)=\frac{1}{2} \int_{-l}^{l}\left(v^{2}+\eta_{\varepsilon}\right)\left(u^{\prime}\right)^{2} \mathrm{~d} x+G_{c} \int_{-l-\delta}^{l+\delta}\left(\frac{(1-v)^{2}}{4 \varepsilon}+\varepsilon\left(v^{\prime}\right)^{2}\right) \mathrm{d} x,
$$

where $\tilde{\Omega}=(-l-\delta, l+\delta)$ for some $\delta>0$. Indeed, a careful analysis of the $\Gamma$-convergence of $E_{\varepsilon}$ reveals that as long as one does not consider interface cracks at $x=-l$ or $x=l$, one can identify 
$\tilde{\Omega}$ and $\Omega$, and consider

$$
E_{\varepsilon}(u, v)=\frac{1}{2} \int_{-l}^{l}\left(v^{2}+\eta_{\varepsilon}\right)\left(u^{\prime}\right)^{2} \mathrm{~d} x+G_{c} \int_{-l}^{l}\left(\frac{(1-v)^{2}}{4 \varepsilon}+\varepsilon\left(v^{\prime}\right)^{2}\right) \mathrm{d} x .
$$

\subsection{Stability analysis}

The first order optimality conditions for the minimizers $u$ and $v$ of $F_{\varepsilon}$ are that for any $(\tilde{u}, \tilde{v}) \in$ $W_{0}^{1,2}(-l, l) \times W^{1,2}(-l, l)$ such that $\tilde{v}^{\prime}(-l)=\tilde{v}^{\prime}(l)=0$, one has

$$
\begin{array}{r}
\int_{-l}^{l}\left(v^{2}+\eta_{\epsilon}\right) u^{\prime} \tilde{u}^{\prime} \mathrm{d} x=0, \\
\int_{-l}^{l}\left(u^{\prime}\right)^{2} v \tilde{v} \mathrm{~d} x+2 G_{c} \int_{-l}^{l}\left(\frac{(v-1) \tilde{v}}{4 \varepsilon}+\varepsilon \tilde{v}^{\prime} v^{\prime}\right) \mathrm{d} x=0 .
\end{array}
$$

The sequence for the upper $\Gamma$-limit in [AT92, Bou98] provides a construction of a pair $\left(u_{\varepsilon}^{f}, v_{\varepsilon}^{f}\right)$ such that $u_{\varepsilon}^{f} \rightarrow u^{f}$ and that 21 are satisfied asymptotically as $\varepsilon \rightarrow 0$. It is also easy to see that the pair

$$
\begin{aligned}
& u_{\varepsilon}^{e}(x)=\frac{t}{l} x, \\
& v_{\varepsilon}^{e}(x)=\frac{G_{c} l^{2}}{G_{c} l^{2}+2 \varepsilon t^{2}}
\end{aligned}
$$

also satisfies both conditions above and that $\left(u_{\varepsilon}^{e}, v_{\varepsilon}^{e}\right) \rightarrow\left(u^{e}, 1\right)$ as $\varepsilon \rightarrow 0$.

A study of the second variation of $F_{\varepsilon}$ around $\left(u_{\varepsilon}^{e}, v_{\varepsilon}^{e}\right)$ will allow us to state the following stability theorem:

THEOREM 5 Given $\varepsilon>0$, there exists a critical load $t_{\varepsilon}^{s}$ such that $\left(u_{\varepsilon}^{e}, v_{\varepsilon}^{e}\right)$ is a saddle point of $F_{\varepsilon}$ if $t \geqslant t_{\varepsilon}^{s}$.

Proof. Consider $(\tilde{u}, \tilde{v}) \in W_{0}^{1,2}(-l, l) \times W^{1,2}(-l, l)$ and $(\alpha, \beta) \in \mathbb{R}^{2}$. Then we have

$$
\begin{aligned}
& F_{\varepsilon}\left(u_{\varepsilon}^{e}+\alpha \tilde{u}, v_{\varepsilon}^{e}+\beta \tilde{v}\right)=F_{\varepsilon}\left(u_{\varepsilon}^{e}, v_{\varepsilon}^{e}\right)+\frac{\alpha^{2}}{2} \int_{-l}^{l}\left(\eta_{\varepsilon}+\left(v_{\varepsilon}^{e}\right)^{2}\right)\left(\tilde{u}^{\prime}\right)^{2} \mathrm{~d} x \\
& +2 \alpha \beta \int_{-l}^{l} u_{\varepsilon}^{e^{\prime}} \tilde{u}^{\prime} v_{\varepsilon}^{e} \tilde{v} \mathrm{~d} x+\beta^{2} \int_{-l}^{l}\left(\frac{\left(u_{\varepsilon}^{e^{\prime}}\right)^{2} \tilde{v}^{2}}{2}+G_{c}\left(\frac{\tilde{v}^{2}}{4 \varepsilon}+\varepsilon\left(\tilde{v}^{\prime}\right)^{2}\right)\right) \mathrm{d} x+o\left(\alpha^{2}, \alpha \beta, \beta^{2}\right)
\end{aligned}
$$

Using the fact that $u_{\varepsilon}^{e}=t x / l$ and that $v_{\varepsilon}^{e}$ is constant, we obtain

$$
\begin{aligned}
F_{\varepsilon}\left(u_{\varepsilon}^{e}+\alpha \tilde{u}, v_{\varepsilon}^{e}+\beta \tilde{v}\right)= & F_{\varepsilon}\left(u_{\varepsilon}^{e}, v_{\varepsilon}^{e}\right)+\alpha^{2} \frac{\eta_{\varepsilon}+\left(v_{\varepsilon}^{e}\right)^{2}}{2} \int_{-l}^{l}\left(\tilde{u}^{\prime}\right)^{2} \mathrm{~d} x+2 \alpha \beta \frac{t}{l} v_{\varepsilon}^{e} \int_{-l}^{l} \tilde{u}^{\prime} \tilde{v} \mathrm{~d} x \\
& +\beta^{2} \frac{t^{2}}{2 l^{2}} \int_{-l}^{l}\left(\tilde{v}^{2}+G_{c}\left(\frac{\tilde{v}^{2}}{4 \varepsilon}+\varepsilon\left(\tilde{v}^{\prime}\right)^{2}\right)\right) \mathrm{d} x+o\left(\alpha^{2}, \alpha \beta, \beta^{2}\right) .
\end{aligned}
$$


Consider now $\tilde{u} \in \mathcal{C}_{0}^{\infty}(-l, l)$ such that $\int_{-l}^{l}\left(\tilde{u}^{\prime}\right)^{2} \mathrm{~d} x=1$, and $\tilde{v}=-\tilde{u}^{\prime}$. Then 25 reduces to

$$
\begin{aligned}
F_{\varepsilon}\left(u_{\varepsilon}^{e}+\alpha \tilde{u}, v_{\varepsilon}^{e}+\beta \tilde{v}\right)= & F_{\varepsilon}\left(u_{\varepsilon}^{e}, v_{\varepsilon}^{e}\right)+\alpha^{2} \frac{\eta_{\varepsilon}+\left(v_{\varepsilon}^{e}\right)^{2}}{2}-2 \alpha \beta \frac{t}{l} v_{\varepsilon}^{e} \\
& +\beta^{2}\left[\frac{t^{2}}{2 l^{2}}+\frac{G_{c} l}{4 \varepsilon}+\varepsilon G_{c} \int_{-l}^{l}\left(\tilde{v}^{\prime}\right)^{2} \mathrm{~d} x\right]+o\left(\alpha^{2}, \alpha \beta, \beta^{2}\right) .
\end{aligned}
$$

The right hand side is minimized when

$$
\alpha=\frac{2 t v_{\varepsilon}^{e}}{l\left(\eta_{\varepsilon}+\left(v_{\varepsilon}^{e}\right)^{2}\right)} \beta
$$

and we obtain

$$
\begin{aligned}
F_{\varepsilon}\left(u_{\varepsilon}^{e}+\frac{2 t v_{\varepsilon}^{e}}{l\left(\eta_{\varepsilon}+\left(v_{\varepsilon}^{e}\right)^{2}\right)} \beta \tilde{u}, v_{\varepsilon}^{e}+\beta \tilde{v}\right)=F_{\varepsilon}\left(u_{\varepsilon}^{e}, v_{\varepsilon}^{e}\right) \\
+\beta^{2}\left[\frac{t^{2}}{2 l^{2}}\left(1-\frac{4\left(v_{\varepsilon}^{e}\right)^{2}}{\eta_{\varepsilon}+\left(v_{\varepsilon}^{e}\right)^{2}}\right)+\frac{G_{c}}{4 \varepsilon}+\varepsilon G_{c} \int_{-l}^{l}\left(\tilde{v}^{\prime}\right)^{2} \mathrm{~d} x\right]+o\left(\beta^{2}\right) .
\end{aligned}
$$

Recalling that $\eta_{\varepsilon} \rightarrow 0$ and $v_{\varepsilon}^{e} \rightarrow 1$ as $\varepsilon \rightarrow 0$, and that $1 \ll l$, we now see that the coefficient of $t^{2}$ becomes negative when $\varepsilon$ is small enough, which in turn means that when $t$ is large enough,

$$
F_{\varepsilon}\left(u_{\varepsilon}^{e}+\frac{2 t v_{\varepsilon}^{e}}{l\left(\eta_{\varepsilon}+\left(v_{\varepsilon}^{e}\right)^{2}\right)} \beta \tilde{u}, v_{\varepsilon}^{e}+\beta \tilde{v}\right)-F_{\varepsilon}\left(u_{\varepsilon}^{e}, v_{\varepsilon}^{e}\right)<0
$$

for small enough $\beta$. This implies that $\left(u_{\varepsilon}^{e}, v_{\varepsilon}^{e}\right)$ is not a local minimum but a saddle point for $F_{\varepsilon}$.

Practically, we see that for large enough loads, the critical point $\left(u_{\varepsilon}^{e}, v_{\varepsilon}^{e}\right)$ loses its stability. For any $v_{0}$, there exists a critical load $\delta_{c}$ such that if $t>\delta_{c}$, then $\left(u_{1}, v_{0}\right)$ is a descent direction for $F_{\varepsilon}\left(u_{\varepsilon}^{e}, v_{\varepsilon}^{e}\right)$. In particular, this implies that for all subsequent iterations of the Alternate Minimizations algorithm, we have $F_{\varepsilon}\left(u_{i}, v_{i}\right)<F_{\varepsilon}\left(u_{\varepsilon}^{e}, v_{\varepsilon}^{e}\right)$. When this happens, the Alternate Minimizations scheme cannot converge towards $\left(u_{\varepsilon}^{e}, v_{\varepsilon}^{e}\right)$, and will converge toward another critical point $\left(u_{\varepsilon}^{c}, v_{\varepsilon}^{c}\right)$. Short of being able to prove that $\left(u_{\varepsilon}^{e}, v_{\varepsilon}^{e}\right)$ and $\left(u_{\varepsilon}^{f}, v_{\varepsilon}^{f}\right)$ are the only critical points of $F_{\varepsilon}$, we cannot ensure that $\left(u_{\varepsilon}^{c}, v_{\varepsilon}^{c}\right)=\left(u_{\varepsilon}^{f}, v_{\varepsilon}^{f}\right)$.

REMARK 6 The construction of the descent direction above does not give a sharp estimate for the critical load $t_{\varepsilon}^{f}$ above which $\left(u_{\varepsilon}^{e}, v_{\varepsilon}^{e}\right)$ becomes a saddle point. This is not an issue in the practical implementation, as it suffices to increase the load until bifurcation toward the "cracked" solution happens, and then use the Backtracking algorithm. Note, however, that since $t_{\varepsilon}^{f} \rightarrow \infty$ as $\varepsilon \rightarrow 0$, one may have to consider very large loads before bifurcation happens. There are several ways to address this issue numerically, including implementing a continuation algorithm on the regularization parameter $\varepsilon$ or adding perturbations to the field $v$. These numerical techniques are being investigated.

\subsection{Numerical results}

In order to illustrate the result of the previous section, we ran several numerical experiments on a two-dimensional beam with $l=5$. In the one we discuss later, the mesh consists of approximately 149,000 linear finite elements and 75,000 nodes (a coarser mesh would lead to similar results). Total 


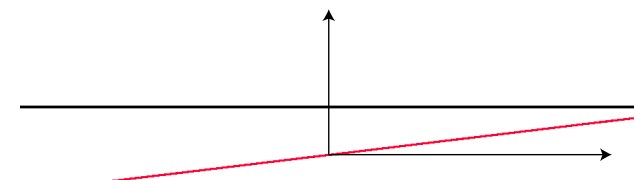

(a) $t \leqslant t_{\varepsilon}^{f}$

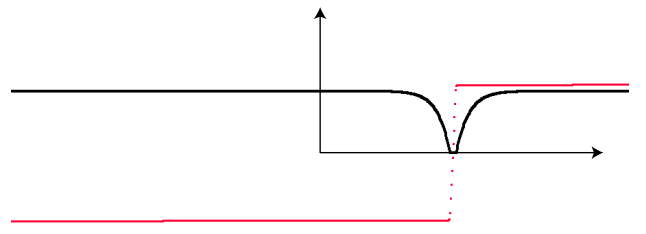

(b) $t \geqslant t_{\varepsilon}^{f}$

FIG. 1. $(u, v)$ profiles.

computation time is less than $1 / 2$ hour, using 32 Intel Xeon $1.8 \mathrm{GHz}$ processors. The computational cost is $1.155 \cdot 10^{12}$ Flops, the mesh size $h=1.5 \cdot 10^{-2}$, the regularization parameter $\varepsilon=8.0$. $10^{-2}$, and $\eta_{\epsilon}$ is $1.0 \cdot 10^{-7}$. We discretized the time interval $(0,10)$ into 201 equi-distributed time steps. The material properties are $G_{c}=1, E=1$, and $v=0$. For this choice of parameters, the critical load upon which total failure of the domain should happen is $t_{c}=\sqrt{5} \simeq 2.2361$. Figure 1 represents the profiles $u_{z}$ and $v_{z}$ corresponding to the uncracked (left) and cracked (right) solutions. Figure 2(a) represents the bulk, surface and total energies as a function of the load, without Backtracking. As expected, the critical failure load is overestimated, with a bifurcation taking place at $t \simeq 7.8$, and the total energy is not monotonic. Figure 2 b) represents the total energy of the system as a function of the iteration number (dashed line) and its theoretical value (solid line). The outcome of the Backtracking algorithm is the following: at first, time steps 0 to 7.8 are computed, and the Alternate Minimizations algorithm fails to bifurcate towards the cracked solution. At $t \simeq$ 7.85, the cracked solution becomes feasible for the Alternate Minimizations algorithm, and the total energy of the system decreases. At this point, condition 18 is not satisfied for all time steps with $t>t_{c}$, and the Backtracking algorithm returns to $t \simeq 2.4$, initializing the Alternate Minimizations algorithm with the cracked solution. The final total energy corresponds to the lower envelope of the dashed curve. The error on the total energy is less than $10 \%$.

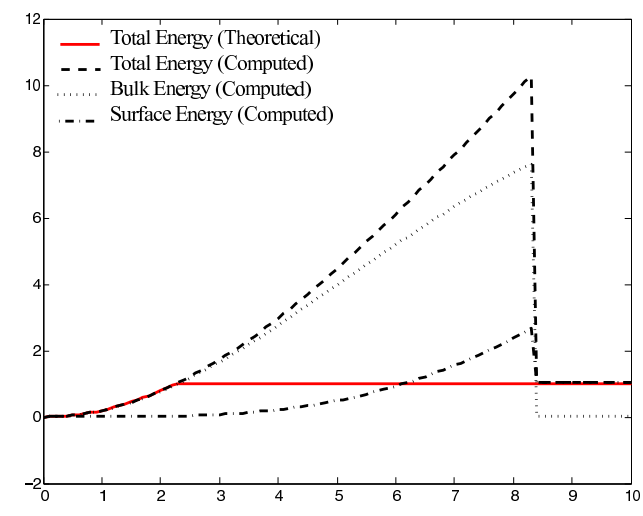

(a) Without Backtracking

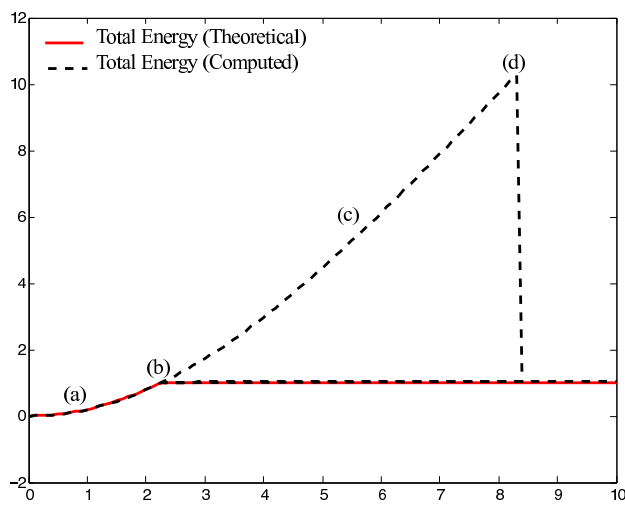

(b) With Backtracking

FIG. 2. Energy as a function of the load for the long beam.

Figure 3 formally illustrates Theorem 5 , and shows how the Backtracking algorithm allows switching from one critical point to the other. It represents a sketch of the shape of the total energy as a function of $(u, v)$ for various loads. The letters (a), (b), (c), (d) correspond to the locations highlighted in Figure 2,b). Figure 3(a) is a sketch of the total energy as a function of $(u, v)$ for a 


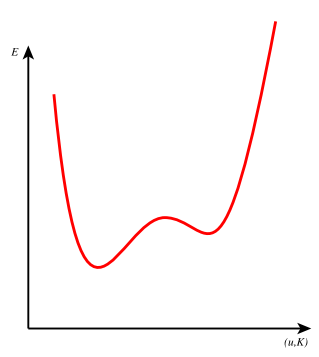

(a) $0<t<t_{c}$

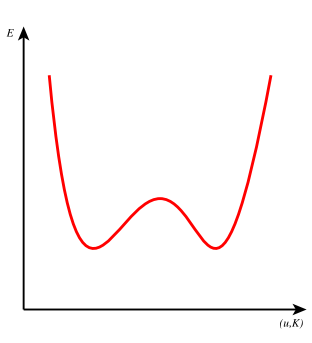

(b) $t=t_{c}$

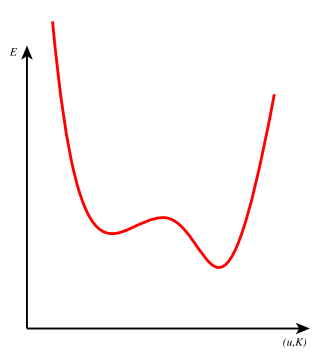

(c) $t_{c}<t<t_{\mathcal{E}}^{f}$

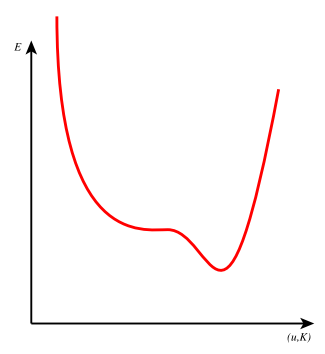

(d) $t \geqslant t_{\varepsilon}^{f}$

FIG. 3. Energy profile as a function of the load.

load $t<t_{c}$. The leftmost well corresponds to the uncracked solution (in this case also the global minimizer) and the right one to the cracked solution. Started from within the left well, the Alternate Minimizations will converge to the uncracked solution. Figure 3 (b) corresponds to the critical load $t=t_{c}$. In Figure 3(c), the load is such that $t_{c}<t<t_{\varepsilon}^{f}$. The global minimum corresponds to the cracked solution, but the outcome of the Alternate Minimizations algorithm will depend on the well in which it is initialized. Finally, Figure 3 (d) corresponds to $t \geqslant t_{\varepsilon}^{f}$. In this case, the Alternate Minimizations will converge to the cracked solution, providing a better initial guess for the time steps $t_{c} \leqslant t \leqslant t_{\varepsilon}^{f}$.

The fact that the numerical scheme eventually converges towards a "cracked" solution may seem counter-intuitive. Indeed, when $u^{\prime}$ and $v$ are constant, the gradient of $F_{\varepsilon}$ with respect to $v$ is constant (up to the discretization error). In a gradient-based algorithm, it would be very difficult to escape from the "uncracked" solution. Alternating full minimization with respect to $u$ and $v$ certainly makes it easier.

It is also worth noticing that in the previous section, we have considered perturbations with norm 1. In the numerical experiments, the discretization, rounding and iterative solver errors play that role. Because of their much smaller magnitude, we do not expect the critical load $t_{\varepsilon}^{f}$ to coincide with the load at which bifurcation happens in the numerical experiments.

\section{Numerical experiments}

\subsection{Revisiting the fiber pullout experiment}

The main contribution of our Backtracking algorithm is to identify crack evolutions which do not satisfy the global necessary condition for optimality (18). Indeed, a quick literature survey reveals that this is a common issue in numerical experiments. In [Neg03, Figure 11], the total energy for a mode-I experiment in planar elasticity is shown to be decreasing for $.26 \leqslant t \leqslant .27$, which is not compatible with (18). Similarly, [BFM00, Section 3.2] presents a traction experiment on a square plate, reinforced at its center by an unbreakable fiber. In Figure 3, the total energy is also decreasing around the critical loads corresponding to brutal evolution, which is consistent with the analysis in the previous section. The same experiment is presented in [DLM07, Figure 7] with a similar outcome.

We re-ran this experiment on a thinner mesh with and without Backtracking algorithm. The domain $\Omega$ corresponds to the black area in Figure 5. The circle in the center of the domain 


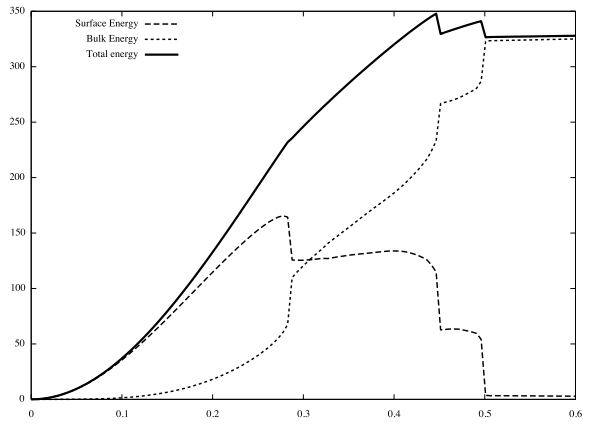

(a) Energies, without Backtracking

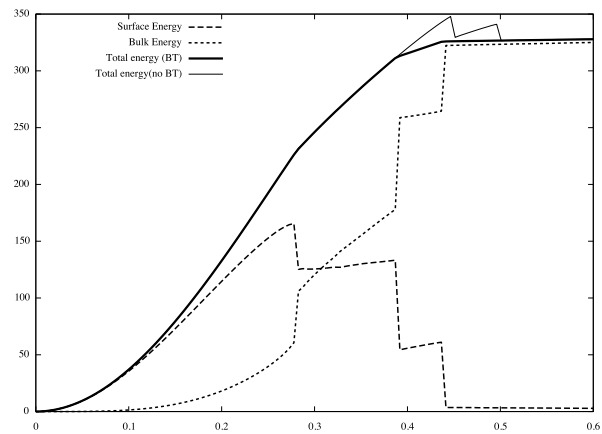

(b) Energies, with Backtracking

FIG. 4. Traction experiment on a fiber-reinforced matrix, energy evolution.

represents a fiber on which homogeneous Dirichlet boundary conditions are applied, while on the upper rectangle, a displacement $u=(0, t)$ is prescribed. The radius of the inclusion is .5 , the length of the square edges 3 , the material properties are set to $E=4,000, v=.2$, and $G_{c}=100$. The mesh consists of approximately 36,000 nodes and 72,000 elements, and we performed 125 load increments for $0 \leqslant t \leqslant .625$. The parameters $\varepsilon$ and $\eta_{\varepsilon}$ are respectively $10^{-1}$ and $10^{-6}$, and the irreversibility threshold is $\alpha_{\varepsilon}=10^{-2}$.

Figure 4(a) represents the evolution of the bulk, surface and total energies as a function of the load when the Backtracking algorithm is not used. It is similar to Figure 3 in [BFM00]. In particular, it is easy to see that condition $[18)$ is violated around $t=.45$ and $t=.5$, which are the critical loads upon which brutal crack propagation takes place. Figure 4(b) correspond to the outcome of the same simulation, using the Backtracking algorithm. The total energy from the previous case is also plotted for comparison. When using the Backtracking algorithm, condition (18) is satisfied for all $t$. The qualitative crack evolution is similar in both cases, and consistent with the description in [BFM00], however, the Backtracking algorithm leads to a better estimate of the critical loads upon which the ligaments fail.

Figure 5 depicts the crack evolution for selected loads. The points where $v>\alpha_{\varepsilon}$ are black, while those with $v \leqslant \alpha_{\varepsilon}$ are white. This post-processing makes the location of the cracks obvious. The original field $v$, which is not plotted here, is still a smooth function.

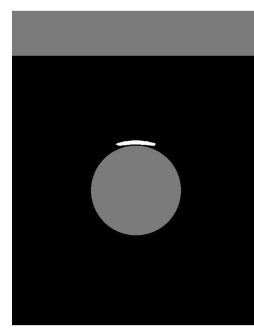

(a) $t \simeq .283$

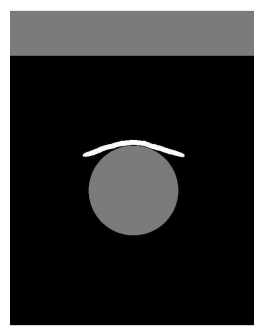

(b) $t \simeq .382$

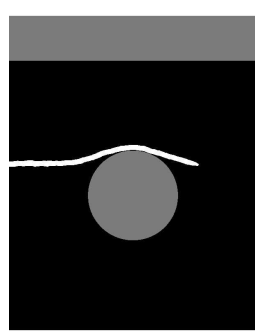

(c) $t \simeq .387$

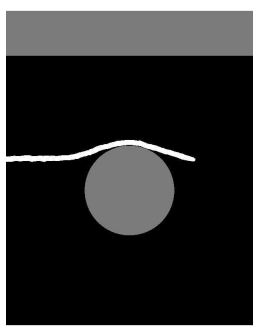

(d) $t \simeq .431$

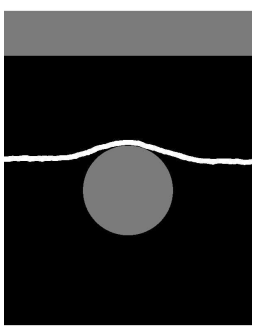

(e) $t \simeq .436$

FIG. 5. Traction experiment on a fiber-reinforced matrix, crack evolution. 


\subsection{Perforated plate under mode-I load}

The last numerical result we present is the simulation of a mode-I traction experiment on a precracked perforated plate. We consider a rectangular domain $\Omega=(-.5, .5) \times(-1,1)$ with a preexisting crack along the segment $-1 \leqslant y \leqslant 0, x=0$, and equi-distributed perforations of diameter .03 (see Figure 6. On the lower edge of $\Omega$, one applies a constant displacement, $u(x, y)=(-t, 0)$ if $x<0$ and $u(x, y)=(t, 0)$ if $x>0$, corresponding to the mode-I opening of the pre-existing crack. The material properties of the samples are $E \simeq 25.2, v \simeq .2857$ (corresponding to $\lambda=12$ and $\mu=9$ ), and $G_{c}=1.0$. Figure 6 represents the deformation of the sample, along with the evolution of the crack, using the Backtracking algorithm. Again, the points where $v>2 \cdot 10^{-2}$ are black, while those with $v \leqslant 2 \cdot 10^{-2}$ white. We ran this computation on a variety of meshes ranging from $h \simeq 10^{-1}$ (30,084 nodes and 15,655 elements) to $h \simeq 3.3 \cdot 10^{-3}$ (134,784 nodes and 265,855 elements), using up to 600 time steps. The unstructured meshes were generated using a DelaunayVoronoy algorithm, and are therefore almost isotropic. The full computation on the largest mesh took 8 hours on 64 processors of NCSA's Teragrid cluster, with a cost of $1.154 \cdot 10^{14}$ Flops.

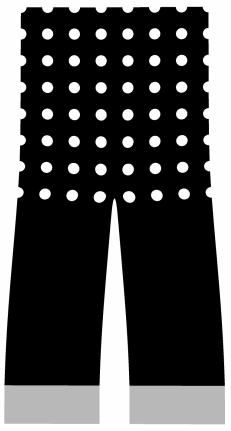

(a) $t \simeq .781$

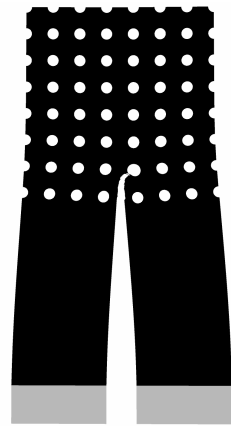

(b) $t \simeq .786$

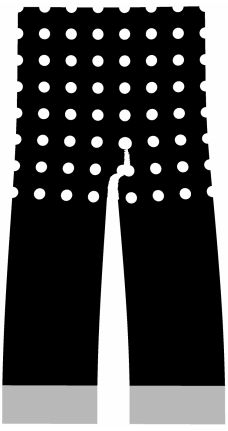

(c) $t \simeq .841$

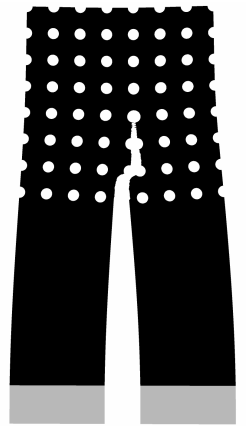

(d) $t \simeq .931$

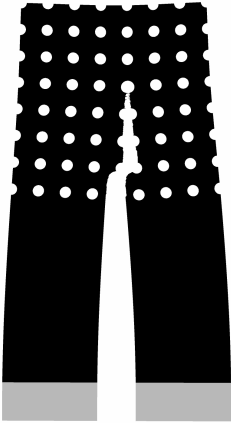

(e) $t \simeq 1.00$

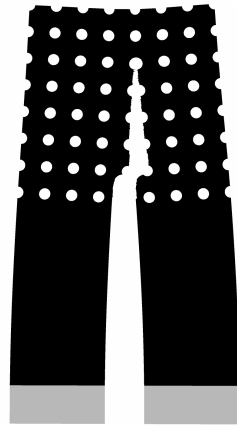

(f) $t \simeq 1.03$

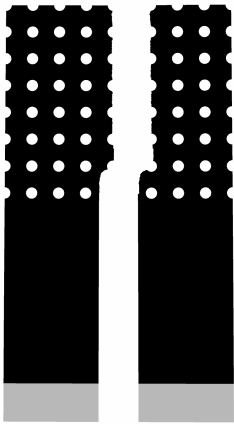

(g) $t \simeq 1.04$

FIG. 6. Perforated plate subject to mode-I traction

Figure 7 represents the evolution of the bulk, surface and total energies as a function of the load (with Backtracking on the left and without on the right), and a comparison of the total energies at the center. 


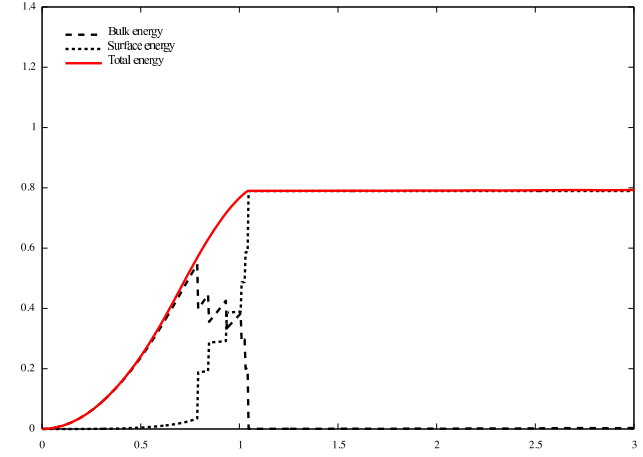

(a) Components of the energy (with Backtracking)

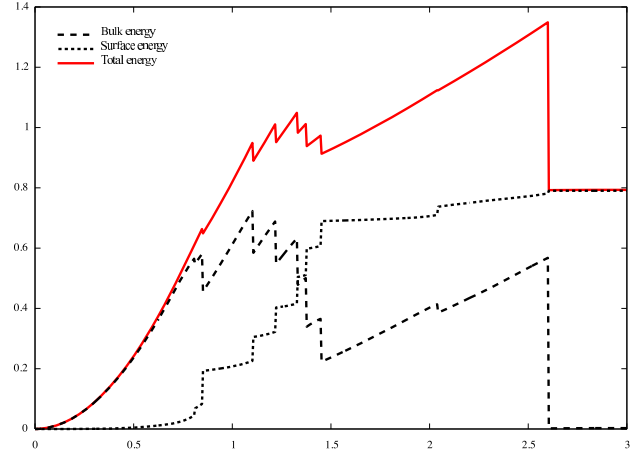

(b) Components of the energy (without Backtracking)

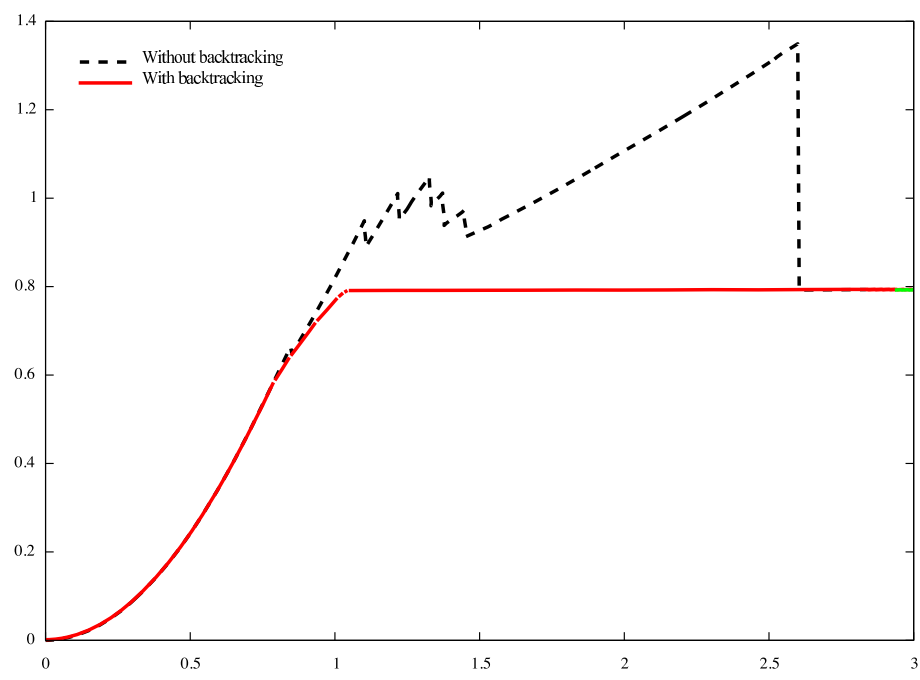

(c) Comparison of the total energies

FIG. 7. Energy as a function of the load for the perforated plate problem.

For loads $0 \leqslant t<.785$, the pre-existing crack does not propagate, and the total energy is equal to the bulk energy (see Figure 6(a)). At a critical load $t \simeq .786$, the crack propagates brutally until it reaches one of the perforations (Figure 6(b)), then remains unchanged again until $t \simeq .841$. At $t \simeq 841$, again it propagates brutally until a second perforation (Figure 6(c)). After that, the crack continues to propagate brutally from one perforation to the next for loads $t \simeq .931$ (Figure 6(d)), $t \simeq 1.00$ (Figure 6(e)), and $t \simeq 1.03$ (Figure 6(f)). Finally, at time $t \simeq 1.04$, the last two links break at the same time, and the sample reaches final failure (Figure6(e)). The brutal behavior of the crack set can be seen from the form of the surface energy in Figure 7 a), which is almost piecewise constant.

In this case again, the Backtracking method leads to a solution with a lower total energy (Figure 7(c)), and to a consistent time evolution, satisfying the growth condition at each time step. More importantly, the qualitative crack behavior is different: using Alternate Minimizations without 
Backtracking, one obtains a smooth crack growth in between the last two holes-compare the slowly increasing surface energy for $2.0 \leqslant t \leqslant 2.5$ in Figure $7(\mathrm{~b})$ to the piecewise constant surface energy in Figure 7(a) Also, the Backtracking leads to an accurate prediction of critical loads inducing crack propagation, while straightforward minimization overestimates them (compare the location of discontinuities in the surface energy in Figures 7(a) and 7(b)].

\section{Acknowledgements}

Support for this work was provided by the Louisiana Board of Regents grant LEQSF (2003-06)-RDA-05 and the National Science Foundation grant DMS-0605320. Parts of the numerical experiments were performed using the National Science Foundation TeraGrid resources provided by NCSA under the Resource Allocation TG-DMS060011N.

The author is deeply indebted to the editor, R. V. Kohn, and the two anonymous reviewers who stepped far beyond their expected roles by providing countless fruitful comments and advice, as well as suggesting several substantial improvements to the original manuscript.

\section{REFERENCES}

[Alb00] Alberti, G. Variational models for phase transitions, an approach via $\Gamma$-convergence. Calculus of Variations and Partial Differential Equations, G. Buttazzo et al. (ed.), Springer (2000), 95-114. Zbl 0957.35017 MR 1757697

[AT90] Ambrosio, L., \& Tortorelli, V. M. Approximation of functionals depending on jumps by elliptic functionals via $\Gamma$-convergence. Comm. Pure Appl. Math. 43 (1990), 999-1036. Zbl 0722.49020 MR 1075076

[AT92] Ambrosio, L., \& Tortorelli, V. M. On the approximation of free discontinuity problems. Boll. Un. Mat. Ital. B (7) 6 (1992), 105-123. Zbl 0776.49029 MR 1164940

[BC94] Bellettini, G., \& Coscia, A. Discrete approximation of a free discontinuity problem. Numer. Funct. Anal. Optim. 15 (1994), 201-224. Zbl 0806.49002 MR 1272202

[BC00] Bourdin, B., \& CHAmbolle, A. Implementation of an adaptive finite-element approximation of the Mumford-Shah functional. Numer. Math. 85 (2000), 609-646. Zbl 0961.65062 MR 1771782

[BFM00] Bourdin, B., FrancFort, G. A., \& MARigo, J.-J. Numerical experiments in revisited brittle fracture. J. Mech. Phys. Solids 48 (2000), 797-826. Zbl 0995.74057 MR 1745759

[BFMar] Bourdin, B., Francfort, G. A., \& Marigo, J.-J. The Variational Approach to Fracture (reprinted from J. Elasticity). Springer, to appear.

[Bou98] Bourdin, B. Une méthode variationnelle en mécanique de la rupture, théorie et applications numériques. Ph.D. thesis, Université Paris Nord, Institut Galilée (1998); http://www.math.lsu. edu/rbourdin.

[Bou99] Bourdin, B. Image segmentation with a finite element method. M2AN Math. Model. Numer. Anal. 33 (1999), 229-244. Zb1 0947.65075 MR 1700033

[Bra02] Braides, A., $\Gamma$-convergence for Beginners. Oxford Lecture Ser. Math. Appl. 22, Oxford Univ. Press, Oxford (2002). Zbl pre01865939 MR 1968440

[CO96] CAMACHO, G. T., \& ORTIZ, M. Computational modelling of impact damage in brittle materials. Int. J. Numer. Meth. Engrg. 33 (1996), 2899-2938. Zbl 0929.74101

[Cha04] Chambolle, A. An approximation result for special functions with bounded variations. J. Math. Pures Appl. 83 (2004), 929-954. Zbl pre02118455 MR 2074682

[Cha05] Chambolle, A. Addendum to "An approximation result for special functions with bounded deformation": the $n$-dimensional case. J. Math. Pures Appl. 84 (2005), 137-145. Zbl pre02118455 MR 2112874 
[CGP05] Chambolle, A., Giacomini, A., \& Ponsiglione, M. Crack initiation in elastic bodies. Arch. Ration. Mech. Anal., 2005, to appear.

[Dal93] Dal Maso, G. An Introduction to $\Gamma$-convergence. Birkhäuser, Boston (1993). Zbl 0816.49001 MR 1201152

[DFT04] Dal Maso, G., Francfort, G. A., \& Toader, M. Quasi-static evolution in brittle fracture: the case of bounded solutions. Calculus of Variations: Topics from the Mathematical Heritage of E. De Giorgi, Quad. Mat. 14, Dept. Mat., Seconda Univ. Napoli, Caserta (2004), 245-266. Zbl pre02159267 MR 2118420

[DLM07] Del Piero, G., LANCIONI, G., \& MARCh, R. A variational model for fracture mechanics: numerical experiments. J. Mech. Phys. Solids, 2007, to appear.

[FL03] FRANCFORT, G. A., \& LARSEN, C. Existence and convergence for quasi-static evolution in brittle fracture. Comm. Pure Appl. Math. 56 (2003), 1465-1500. Zbl 1068.74056 MR 1988896

[FM98] FRANCFORT, G. A., \& MARIGO, J.-J. Revisiting brittle fracture as an energy minimization problem. J. Mech. Phys. Solids 46 (1998), 1319-1342. Zbl 0966.74060 MR 1633984

[Gia05] Giacomini, A. Ambrosio-Tortorelli approximation of quasi-static evolution of brittle fractures. Calc. Var. Partial Differential Equations 22 (2005), 129-172. Zbl 1068.35189| MR 2106765

[GP03] Giacomini, A., \& Ponsiglione, M. A discontinuous finite element approximation of quasistatic growth of brittle fractures. Numer. Funct. Anal. Optim. 24 (2003), 813-850. Zbl 1046.74048 MR 2011593

[Gri21] Griffith, A. A. The phenomena of rupture and flow in solids. Philos. Trans. Roy. Soc. London Ser. A 221 (1921), 163-198.

[MM77a] Modica, L., \& Mortola, S. Il limite nella $\Gamma$-convergenza di una famiglia di funzionali ellittici. Boll. Un. Mat. Ital. A (5) 14 (1977), 526-529. Zbl 0364.49006 MR 0473971

[MM77b] Modica, L., \& Mortola, S. Un esempio di $\Gamma^{-}$-convergenza. Boll. Un. Mat. Ital. B (5) 14 (1977), 285-299. Zbl 0356.49008 MR 0445362

[MDB99] Mö̈s, N., Dolbow, J., \& Belytschko, T. A finite element method for crack growth without remeshing. Int. J. Numer. Meth. Engrg. 46 (1999), 131-150. Zbl 0955.74066

[Neg03] NeGRI, M. A finite element approximation of the Griffith model in fracture mechanics. Numer. Math. 95 (2003), 653-687. Zbl 1068.74080 MR 2013123

[Ton05] Tonegawa, Y. On stable critical points for a singular perturbation problem. Comm. Anal. Geom. 13 (2005), 439-459. Zbl pre05000499 MR 2154826

[XN94] XU, X. P., \& Needleman, A. Numerical simulations of fast crack-growth in brittle solids. Internat. J. Solids Structures 42 (1994), 1397-1434. Zbl 0825.73579 